\title{
High JAG1 expression in adrenocortical carcinomas is associated with better prognosis
}

${ }^{1}$ Endocrine and Diabetes Unit, Dpt. of Internal Medicine I, University Hospital of Wuerzburg, Germany

${ }^{2}$ Institute of Pathology, University of Wuerzburg, Germany

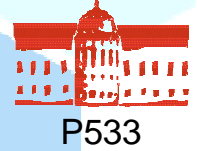

BACKGROUND: Adrenocortical tumors consist of frequent benign adenomas (ACA) and rare highly malignant carcinomas (ACC) with a still incompletely understood pathogenesis.

Dysregulation of the Notch signalling pathway is implicated in several cancers with oncogenic or tumor suppressor functions. Up-regulation of JAG1, a ligand of Notch receptor and a target gene for Notch and $\beta$-catenin pathway (Fig 1), has been reported to enhance cell proliferation in ACC (Ref 1), but no specific data on Notch1 pathway activation or JAG1 protein expression are available.

\section{Fig. 1}

Schematic representation of the Notch signaling Pathway (Ref 2).

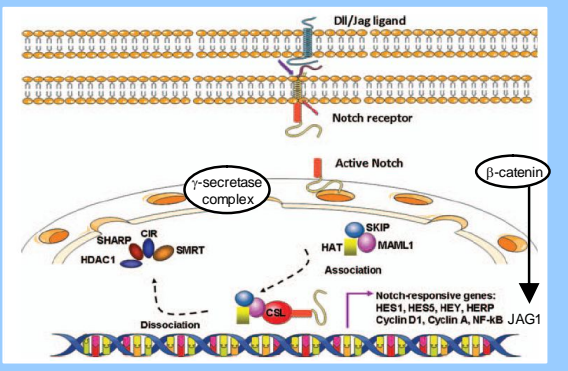

\section{METHODS:}

mRNA expression: NOTCH1, JAG1, and two specific target genes of Notch pathway (HES1 and HEY2) were evaluated in 49 fresh frozen samples (13 normal adrenal glands=NA, 17 ACA, and 19 ACC) by quantitative real-time PCR.

JAG1 protein expression was investigated in 203 tissues on standard paraffin slides or tissue microarrays (7 NA and 196 adrenocortical tumors, $T a b \quad 1)$ by immunohistochemistry (monoclonal anti-rabbit Ab, Lifespan Bioscience, 1:300). Immunostaining was evaluated according to the $\mathrm{H}$-score. The correlation between JAG1 expression and clinical or histopathological parameters was also investigated in ACC.

\begin{tabular}{|l|c|c|c|}
\hline & ACA ( $\mathbf{n}=25)$ & ACC $(\mathbf{n}=\mathbf{1 7 1})$ & P \\
\hline F/M & $17 / 8$ & $116 / 55$ & NS \\
\hline Age (yrs) - median & 47 & 50 & NS \\
\hline Tumor size (cm) - median & 2.8 & 9.7 & $<0.05$ \\
\hline Steroid secretion (n) & & & NS \\
only cortisol/only aldosterone & $12 / 6$ & $30 / 6$ & \\
mixed & - & 26 & \\
inactive & 7 & 23 & \\
not known & - & 86 & \\
\hline
\end{tabular}

Tab. 1 Clinical data in the subgroups of ACAs and ACCs.

\section{RESULTS:}

mRNA expression: NOTCH1 levels were similar in NA and in tumors. JAG1 and HES1 were slightly higher in ACC than in ACA, but HEY2 was significantly higher in ACC (Fig 2).

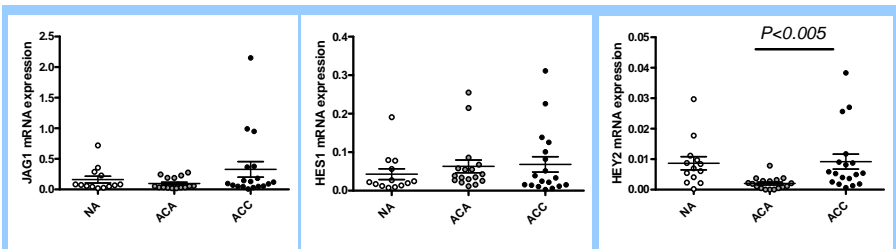

Fig. 2 Relative mRNA expression of JAG1, HES1, and HEY2 in normal adrenal glands (NA), adenomas (ACA) and carcinomas (ACC)
No significant correlations were observed between NOTCH1, JAG1, HES1, and HEY1 mRNA levels and clinical or histopathological data.

JAG1 protein expression: JAG1 staining was often inhomogeneous (percentage of positive cells ranging from $15 \%$ to $90 \%$, Fig 3).

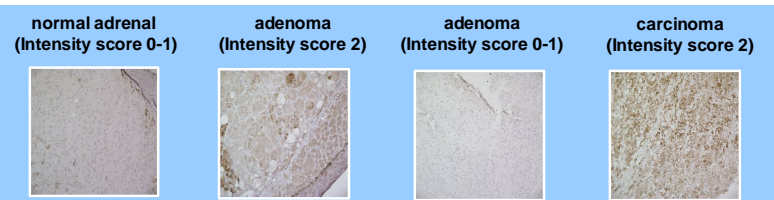

Fig. 3 Different examples of JAG1 immunostaining in normal and neoplastic adrenal glands.

JAG1 protein expression was absent or very low (H-score $\leq 1)$ in $72 \%$ of NA and in $61 \%$ of ACAs, but significantly higher in the ACCs (H-score $>1$ in $73 \%$ of ACCs, $P<0.005$, Fig 4A).

In the ACC group ( $n=126$ patients who underwent first surgery), JAG1 expression was higher in patients with early ENSAT tumor stages than in those with metastatic disease (Fig 4B). No other significant correlations were observed between JAG1 levels and clinical or histopathological parameters.

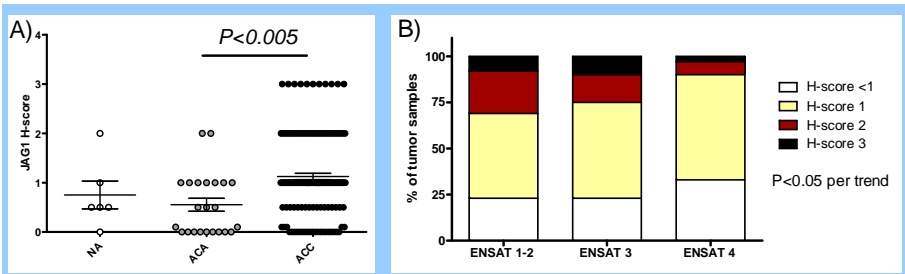

Fig. 4 JAG1 protein expression in normal and neoplastic adrenal glands evaluated as $\mathrm{H}$-score.

Interestingly, high JAG1 expression was significantly associated with a longer overall and disease free survival (Fig $5 \boldsymbol{A}$ and $\boldsymbol{B}$ ). At multivariate analysis including the ENSAT stage, JAG1 maintained its independent impact on overall survival $(P=0.007$, $\mathrm{HR}=0.64,25 \% \mathrm{Cl}: 0.46-0.89)$.

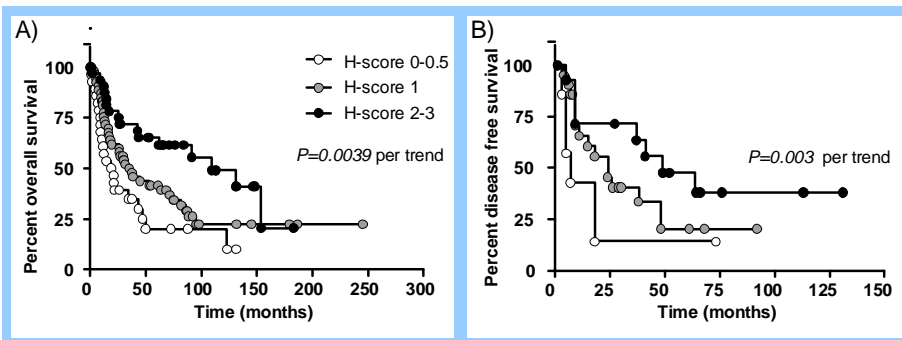

Fig. 5 Impact of JAG1 protein expression on overall survival (A, $n=126)$ and disease free survival $(B, n=45)$ in patients with $A C C$.

\section{CONCLUSION:}

- Notch1 signaling pathway activation might be involved in adrenocortical tumor progression and needs to be further investigated.

- High JAG1 expression is associated with a better clinical outcome in ACC and might represent a new favorable prognostic marker.

\section{References:}

- Simon D, Giordano TJ, Hammer G. Upregulated JAG1 Enhances Cell Proliferation in Adrenocortical Carcinoma. Clin Cancer Res 2012

Yin L, Velazquez OC, Liu ZJ. Notch signaling: Emerging molecular targets for cancer therapy. Biochem Pharmacol. 2010 